\title{
Acute-phase proteins, oxidative stress and antioxidant defense in crib-biting horses
}

Arash Omidi a, ${ }^{*}$, Saeede Vakili a, Saeed Nazifi b, Matthew O. Parker c

a Department of Animal Health Management, School of Veterinary Medicine, Shiraz University, Shiraz, Iran

b Department of Clinical Studies, School of Veterinary Medicine, Shiraz University, Shiraz, Iran

cSchool of Health Sciences and Social Work, University of Portsmouth, Portsmouth, UK

*Corresponding author: Arash Omidi, Associate Professor, Animal Health Management Department, School of Veterinary Medicine, Shiraz University, Shiraz, Iran

Email: aomidi@shirazu.ac.ir

Tel: +987136138745 


\begin{abstract}
Crib-biting is a common equine stereotypic behavior that has links to health problems and poor welfare. There is evidence that crib-biters may be more sensitive to environmental stressors, and the aim of the present study was to test the hypothesis that crib-biting behavior in horses has a relationship with oxidative stress, antioxidant defense or inflammatory proteins, as is observed in humans with various affective disorders such as depression and anxiety. Ten crib-biting horses and ten age- and sex- matched healthy horses were used. Vital signs (Heart rates, respiratory rates and temperature) and blood samples were taken in three conditions; basal condition for crib-biters (no stereotypic behavior observed for at least $30 \mathrm{~min}$ ); Crib-biters during or directly after crib-biting periods ('acute crib-biting' = crib-biting for at least 15 minutes with no interruption longer than 2 minutes); non-cribbiting horses (control). Comparisons were made between crib-biters and controls, and between crib-biters' basal and crib-biting values. No changes were observed in the vital signs. Total antioxidant capacity (TAC) activity was significantly decreased in crib-biters during the basal measurements relative to controls, and this was significantly decreased again during an episode of crib-biting. Similar differences to those observed in TAC were also observed for the antioxidant enzymes superoxide dismutase (SOD), Glutathione peroxidase (GPx) and catalase (CAT). Collectively, our data suggest that the antioxidant levels and antioxidant defenses against lipid peroxidation are reduced in crib-biters, suggesting that oxidative stress plays a role in the pathophysiology of crib biting. In addition, our data suggest that further study on the role of oxidative stress in crib biting might be of benefit, particularly in the search for veterinary treatments or interventions for crib biting horses.
\end{abstract}

Keywords: Acute-Phase Proteins, Antioxidants, Behaviour, Horses, Oxidative Stress, crib-biting.

\title{
1. Introduction
}

Stereotypic behavior is repetitive, compulsive behavior that is observed in a number of human neuropsychiatric or neurodevelopmental disorders that appears to result from dysfunction of the basal ganglia and associated neural circuits (Luescher et al., 1998; McBride and Parker, 2015). Over the past decade, stereotypic behavior in horses, and particularly crib-biting, has received considerable attention in the scientific literature (Cooper and Albentosa, 2005; McBride and Hemmings, 2009; Sarrafchi and Blokhuis, 2013). Crib-biting is an oral based behavior characterized by grasping a fixed object with incisor teeth and engulfing air with an audible grunt (McGreevy et al., 1995). Recently it has become 
apparent that horses that display crib-biting are more stress sensitive, physiologically and psychologically less flexible, and their capacity for operant learning is diminished (Freymond et al., 2015; Nagy et al., 2010; Parker et al., 2009; Parker et al., 2008a). In addition, some studies suggest that crib-biting is associated with altered neurophysiological function [McBride and Hemmings, 2009; Sarrafchi and Blokhuis, 2013).

Crib-biting has important consequences for equine health and welfare (e.g., as a risk factor for epiploic foramen entrapment colic, Hillyer et al., 2002; Archer et al., 2004) and its mechanism of formation requires more investigation. Although there is evidence that 'chronic stress' plays a role in the development of cribbiting (Bachmann et al., 2003; Parker et al., 2008b), the mechanisms by which this change occurs is far from clear. Oxidative stress has been implicated in the pathogenesis of various diseases, and may be a common mechanism in the pathophysiology of many major psychiatric disorders in humans such as depression (Cumurcu et al., 2009; Sarandol et al., 2007) and pathological anxiety (Bouayed et al., 2009). During the production of ATP, oxidative phosphorylation results in free-radical formation, including reactive oxygen species (ROS) such as hydrogen peroxide (H2O2), superoxide radical O2- and hydroxyl radical [OH-]. These increases in free-radicals are mediated by antioxidant responses in the organism to maintain the prooxidant/antioxidant homeostasis. Owing to its large oxygen consumption, the brain is relatively more vulnerable to oxidative damage than other organs (Smith et al., 1991; Love, 1999; Ng et al., 2008). Although the direction of the cause and effect relationship is not firmly established (Andersen, 2004), the results of several studies suggest that lower antioxidant defenses against lipid peroxidation exist in patients with depression and free radicals are involved in the biochemical mechanisms underlying neuropsychiatric disorders (Maurya et al., 2016; Ozcan et al., 2004).

In addition to the link to oxidative stress, it is becoming increasingly clear that inflammation may play an important role in the pathophysiology of depression (Miller and Raison, 2016; Raison et al., 2006 and 2011). Initially, links between affective disorder and inflammation were thought to result from chronic stress causing immunosuppression; however, recent evidence has emerged that instead, depression (and other neuropsychiatric disorders) might be the result of innate immune responses (Jones and Thomsen, 2013; Pace et al., 2006). This has led to the intriguing hypothesis that neuropsychiatric disorders may be caused by alterations in the immunological response to stress (Jones and Thomsen, 2013). Owing to the putative role of stress in the development of stereotypic behaviours such as crib-biting (Parker et al., 2008b; Bachmann et al., 2003) and links between the pathophysiology of stereotypic behaviour and human neuropsychiatric disorders (such as 
affective disorders, compulsive disorders, etc.; McBride and Parker, 2015; Cabib et al., 1998) it is possible that crib biting may have a similar aetiology.

The aim of the present study was to examine whether crib-biting shares characteristics with human neuropsychiatric disorders by carrying out a comprehensive assessment of the physiological correlates of cribbiters both during an acute crib-biting episode and during resting. Specifically, we tested the hypothesis that crib-biting was related to oxidative stress and chronic inflammation. We approached this by assessing the changes in several widely used physiological markers linked to oxidative stress . These markers included Albumin (ALB), which is used in extracellular antioxidant defenses (Witko-Sarsat et al., 1996), Serum Total protein (TP), which is known to reduce following oxidative damage (Erel, 2004), and Globulin (GLB) (Wickens et al., 1983). Acute-phase proteins (APPs) are a class of proteins whose plasma concentrations increase (positive APPs) or decrease (negative APPs) in response to infections and injuries involving inflammation (Ceciliani et al., 2002). Previous work revealed that APPs such as Heptoglobin (Hp), Serum amyloid-A (SAA) and Alpha -1- acid glycoprotein (AGP) will increase in concentration multifold following inflammation and infectious diseases, or stress (Fournier et al., 2000; Murata et al., 2004). Thus we also tested for SAA, AGP, and Hp. Finally, we tested Malondialdehyde (MDA) as a measure of lipid peroxidation (Del Rio et al., 2005), serum total antioxidant capacity (TAC) (Sharma et al., 1999), and levels of antioxidant enzymes Glutathione peroxidase (GPx), superoxide dismutase (SOD), and catalase (CAT) (Armstrong and Brown, 1994).

\section{Materials and methods}

\subsection{Animal ethics}

This study was conducted in November-December 2015 in the Fars province, Iran, around the Shiraz city with the approval of the State Committee on Animal Ethics, Shiraz University, Shiraz, Iran. Recommendations of European Council Directive 86/609/EC (1986) were followed, regarding the guidelines for the protection of animals used for experimental purposes. All experiments were agreed by the Shiraz University Animal Care and Use Committee. All blood sampling was carried out by a fully qualified veterinarian.

2.2.

\section{Animals}

\section{[Table 1 here]}

Table 1 displays the individual characteristics of the sample horses. Ten crib-biting horses and ten sex- and breed- 
matched controls were used. All controls were also age matched as much as possible. All crib-biting horses were current, established crib-biters ( $>1$ year) based on owner reports (full history unclear for all horses). The animals were single housed in conventional horse boxes in different riding stables in the surrounding of Shiraz. All feeding was similar, with crib biters and controls having concentrate in their daily ration. Although we did not directly test the frequency or duration of crib biting, it was noted that handling of the horses did increase the likelihood of crib biting.

\subsection{Study protocol}

To minimize influences of circadian changes, all studies were carried out between 0930 and $1130 \mathrm{~h}$. The vital signs of crib-biting horses were evaluated in their home boxes (Heart rates, respiratory rates and temperature) before blood sampling. Blood samples were taken from all horses in the following conditions: crib-biters [basal]: basal conditions of crib- biting horses (no stereotypic behavior for at least $30 \mathrm{~min}$ ); crib-biters [acute-crib-biting]: during or immediately after crib-biting periods (crib-biting for at least 15 minutes with no interruption longer than 2 minutes); control: non-crib-biting, healthy horses. Blood samples were obtained by jugular venipuncture into plain tubes and vacationers containing EDTA (in a 10:1 ratio) as an anticoagulant.

\subsection{Biochemical analysis}

After centrifugation of blood at $750 \mathrm{~g}$ for $15 \mathrm{~min}$ at room temperature, serum or plasma was separated and stored at $-80{ }^{\circ} \mathrm{C}$ until analysis. The samples with hemolysis were discarded.

\subsubsection{Total protein, albumin and globulin measurements}

Serum was analysed for total protein (TP) by Biuret method (Commercial kit; Pars Azmoon, Tehran, Iran), albumin (ALB) by the bromocresol green method (Commercial kit; Pars Azmoon, Tehran, Iran). Serum total globulin (GLB) was

determined as the difference between serum TP and ALB. Biochemical analyses were measured using a standard autoanalyser with veterinary software (Cobas-Mira, ABX-Diagnostics, Japan).

\subsection{2. $\quad$ Acute phase proteins (Serum amyloid A, Alpha-1-acid glycoprotein and Haptoglobin) determination}

Serum amyloid A (SAA) was measured by a solid phase sandwich-ELISA method with a sensitivity of $0.962 \mathrm{ng} / \mathrm{ml}$ (horse SAA Elisa Kit, Cusabio Biotech Company, Wuhan, China). Alpha-1-acid glycoprotein (AGP) was measured using horse ELISA Kit (My Bio Source Company, USA) with a sensitivity of $1.706 \mathrm{ng} / \mathrm{ml}$. Haptoglobin (Hp) was 
measured using a commercial kit based on prevention of the peroxidase activity of hemoglobin, which is directly proportional to the amount of $\mathrm{Hp}$. The analytical sensitivity of this test in serum has been determined as 0.0156 $\mathrm{mg} / \mathrm{mL}$ for Hp by the manufacturer (Tridelta Development Plc, Wicklow, Ireland).

\subsubsection{Measurement of malondialdehyde (MDA)}

To evaluate lipid peroxidation in serum, a modified HPLC method was used based on the reaction of MDA with thiobarbituric acid (TBA), to form a colored MDA-TBA adduct (Lykkesfeldt, 2001). $40 \mu \mathrm{L}$ of sample was diluted with $100 \mu \mathrm{L}$ of $\mathrm{H} 2 \mathrm{O}$ and mixed with $20 \mu \mathrm{L}$ of $2.8 \mathrm{mmol} / \mathrm{L}$ butylated hydroxyl toluene (BHT) in ethanol, $40 \mu \mathrm{L}$ of $81 \mathrm{~g} / \mathrm{L}$ sodium dodecyl sulfate and $600 \mu \mathrm{L}$ of TBA reagent $(8 \mathrm{~g} / \mathrm{L}$ TBA diluted 1:1 with $200 \mathrm{ml} / \mathrm{L}$ acetic acid adjusted to $\mathrm{pH} 3.5$ with $\mathrm{NaOH})$. The mixture was immediately heated $\left(60 \mathrm{~min}\right.$ at $\left.95^{\circ} \mathrm{C}\right)$ and cooled with running water; $200 \mu \mathrm{L}$ of $\mathrm{H} 2 \mathrm{O}$ and $1000 \mu \mathrm{L}$ of butanol-pyridine (15:1, v/v) were then added. After vigorous mixing, the organic layer was separated by centrifugation ( $3 \mathrm{~min}$ at $16,000 \mathrm{~g}$ ). The supernatant was analyzed on a UV-visible spectrophotometer fitted with an $80 \mu \mathrm{L}$ flow cell. The absorbance was measured at $532 \mathrm{~nm}$ (the mobile phase consisted of $300 \mathrm{~mL} / \mathrm{L}$ methanol in $50 \mathrm{mmol} / \mathrm{L}$ potassium di hydrogen phosphate buffer, $\mathrm{pH}$ 7.0). 1, 1, 3, 3tetraethoxypropane was used as a standard, and MDA-TBA reactive substances' values were expressed as MDA mili-mole per liter (mmol/L). The HPLC system consisted of a solvent delivery pump (JASCO 980-PU, Tokyo, Japan), a reversed-phase column (Luna C18, $250 \mathrm{~mm} \times 4.6 \mathrm{~mm}$, Phenomenex, CA, USA), and a UV-Vis detector (Jasco, UV-975, Tokyo, Japan) operated at $532 \mathrm{~nm}$.

\subsubsection{Measurement of total antioxidant capacity (TAC)}

Determination of total antioxidant capacity (TAC) in serum was ascertained by a commercial kit (Labor Diagnostika Nord (LDN) Com, Nordhorn, Germany). The change in color was measured colorimetrically at 450 $\mathrm{nm}$ and expressed as mili moles per liter.

2.4.5.

Measurement of enzyme activities (superoxide dismutase (SOD), glutathione peroxidase (GPX) and catalase (CAT))

The activity of SOD was measured with a commercial kit (RANSOD kit, Randox Com, UK). According to the manufacturer's manual, $0.5 \mathrm{ml}$ of whole blood was centrifuged for 10 minutes at $700 \mathrm{~g}$ and the plasma was aspirated off. The erythrocytes were washed four times with $3 \mathrm{ml}$ of $0.9 \% \mathrm{NaCl}$ solution and were centrifuged each time to separate the supernatant. The washed erythrocytes were diluted to $2 \mathrm{ml}$ with cold redistilled water. After thorough mixing the lysate was diluted with $0.01 \mathrm{~mol} / 1$ phosphate buffer $(\mathrm{pH}=7)$ with a final dilution factor of 200. The reagents were then added to the diluted samples. In this method, xanthine and xanthine oxidase are 
employed to generate superoxide radicals, which react with 2-(4-iodophenyl)-3-(4-nitrophenol)-5phenyltetrazolium chloride (INT) to form a red formazan dye. The activity of SOD in hemolysate was determined by the degree of inhibition of this reaction as one unit of SOD corresponded to $50 \%$ inhibition of INT reduction under assay condition. Finally, the enzyme activity was expressed as units/g of hemoglobin. The activity of GPx was measured by a commercial kit (RANSEL kit, Randox Com, UK) based on established protocols (Paglia and Valentine, 1967). According to the manual, $0.05 \mathrm{ml}$ of whole blood was diluted and incubated with $3 \mathrm{ml}$ of a diluting agent to form the hemolysate. The GPx present in the hemolysate catalyses the oxidation of glutathione (GSH) by cumene hydroperoxide. In the presence of glutathione reductase and NADPH, the oxidized glutathione is immediately converted to the reduced form with a concomitant oxidation of NADPH to NADP+. The absorbance was measured at $340 \mathrm{~nm}$ and the enzyme activity was expressed as units/g of hemoglobin. The activity of catalase was determined with the commercial catalase assay kit (Oxford Biomedical Research, Inc., USA) based on the colorimetric method, described in Slaughter and O'brien (2000) and the activities of the enzymes were expressed as $\mathrm{U} / \mathrm{g}$ of hemoglobin.

\subsection{Statistical Analysis}

All descriptive statistics are reported as mean \pm SD. Data were analyzed using IBM SPSS Statistics Version 22.0 for Macintosh. Comparison of physiological parameters was carried out by fitting data to random intercept linear mixed effects models. The fixed factor for all models was 'condition', and this had three levels (control; cribbiters 'basal'; crib- biters 'acute crib-biting'). To account for non-independence of the two levels of crib-biting (acute, basal), we included subject ID as a random effect. Owing to the large numbers of dependent variables measured, we adopted a penalized alpha value of $P \leq 0.01$ for all models. Denominator degrees of freedom were estimated by the Satterthwaite approximation. Post-hoc comparisons were carried out with respect to Bonferroni adjusted alpha values. Finally, Pearson's correlation coefficients were used to examine intercorrelations of all physiological parameters (two-tailed significance tests; alpha level: $P \leq 0.05$ ).

\section{Results}

\section{[Table 2 here]}

A summary of vital signs and age in evaluated horses is shown in Table 2. All data were fitted to random intercept linear mixed effects models. There were no significant differences between the conditions for any of the measures 
parameters.

\section{[Table 3 here]}

Table 3 displays the values for all measured physiological parameters for the control, crib-biters [basal] and cribbiters [acute crib-biting] conditions. Random intercept linear mixed effects models revealed no significant main effects of Condition for TB $[F<1]$, ALB $[F<1]$, $\operatorname{GLB}[F<1]$, SAA $[F(2,13)=2.28, P=0.14]$, AGP $[F<1]$, $\mathrm{Hp}[F<1]$, or MDA $[F(2,24)=2.5, P=0.1]$. However, there were main effects for TAC $[F(2,27)=38.43, P<$ 0.001: control $>$ crib- biters (basal) $>$ crib-biters (acute crib-biting), Bonferroni-corrected $P \mathrm{~s}<0.01], \operatorname{SOD}[F(2$, $13)=141.73, P<0.001$ : control $>$ crib-biters (basal) $>$ crib-biters (acute crib-biting), Bonferroni-corrected $P$ s $<$ 0.01], GPx $[F(2,19)=36.26, P<0.001$ : control $>$ crib-biters (basal) $>$ crib-biters (acute crib-biting), Bonferronicorrected $P \mathrm{~s}<0.01]$, and CAT $[F(2,21)=147.56, P<0.001$ : control $>$ crib-biters (basal) $>$ crib-biters (acute cribbiting), Bonferroni-corrected $\left.P_{\mathrm{s}}<0.01\right]$.

\section{[Table 4 here]}

Table 4 displays the intercorrelations between the physiological parameters for all horses. Strong positive correlations were found between TAC, SOD, GPx and CAT. There was also a strong positive correlation between TP and GLB and moderate positive correlations between TP and AGP, and GLB and AGP. Finally, there was a moderate negative correlation between SAA and TAC. There were no other significant intercorrelations between the physiological parameters.

\section{Discussion}

The aim of this study was to test the hypothesis that crib-biting behavior has a relationship with oxidative stress, antioxidant defense or inflammatory proteins. We found partial support for this hypothesis, with crib-biters showing lower basal levels of antioxidant enzymes compared to controls, levels which reduced further during an acute episode of crib-biting. There were some inconsistencies, however, with markers of lipid peroxidation (MDA) being unchanged in crib biters.

TAC, as well as levels of the antioxidant enzymes SOD, GPx and CAT, were all significantly decreased in crib- 
biting horses at rest, and further decreased during an acute phase of crib-biting, indicating that crib biting is associated with reduction in antioxidant capacity. The function of TAC is to assess the capacity of known and unknown antioxidants and their synergistic interaction by monitoring the cumulative action of all the antioxidants present in plasma and body fluids, and TAC will reduce during oxidative stress (Serafini and Del Rio, 2004). Owing to its high metabolic rate and relatively low capacity for cell regeneration, the brain is particularly susceptible to the effects of oxidative stress (Andersen, 2004). There is extensive and growing evidence that increases in free radicals and reduction in antioxidant defenses have a deleterious effect on neural tissue and appear to be fundamentally important in the pathogenesis of neuropsychiatric disorders (Halliwell and Gutteridge, 1990; $\mathrm{Ng}$ et al., 2001). Our evidence does suggest, therefore, that oxidative stress may be involved with the pathophysiology of crib biting. What is not clear, however, is the direction of causation; i.e., is it crib-biting or an additional factor that is causing both physiological and the behavioral effects (for example, psychological stress). Alternatively, it may be that crib-biting represents a behavioral response to higher oxidative stress/reduced antioxidant capacity; however, there is not yet any evidence that crib-biting plays a homoeostatic role in this context.

Not all our markers of oxidative stress were associated with crib biting. For example, in the present study, APPs were unchanged according to crib-biting condition. The APPs in equids are not widely described. The host's body responds to inflammation by producing some APPs such as Hp, SAA and AGP by the liver (Eckersall and Bell, 2010; Bachmann et al., 2003). Without further investigation of APPs in equids, the reasons for these measures not being observed as different in the crib biters is not altogether clear. However, there were intercorrelations between TP and AGP, as well as GLB and AGP. These were independent of crib biting, and may be interesting to examine in future studies in terms of characterizing APPs in equids. It is of also potentially of interest that there were no signs here of inflammation, in particular give the strong links between gastric ulceration, stress and crib biting previously discussed (Nicol et al., 2002; Moeller et al., 2008). The pathophysiology of psychological/physiological stress-induced gastric ulcers has been linked to the effects of oxidative damage; in particular, the hydroxyl radical $(\mathrm{OH} \bullet)$ and associated damage to the gastric mucosa. As we found no changes in APPs in this study, there is no evidence that the crib biters here were experiencing inflammation.

In addition to our observations concerning the antioxidant enzymes, MDA is an indicator of lipid peroxidation and a biomarker for oxidative stress (Lykkesfeldt, 2001). We therefore hypothesized that if crib biting was linked to increased oxidative stress, we would see an increase in MDA. Although in our study we did not find a significant 
increase in MDA levels in the crib biters, we observed a linear trend towards an increase in the concentration of MDA in crib-biting horses. Oxidative stress is a major contributor to the etiology of depression (Dadheech et al., 2006; Maes et al., 2011). In fact, raised levels of MDA due to increased generation of ROS are observed in severe neuropsychiatric disorders in humans (Bilici et al., 2001; Gałecki et al., 2009). The present study may have been limited by our relatively small sample size, but also MDA levels in human neuropsychiatric disorders have often been assessed after many years of the patients suffering with the disorder (Bilici et al., 2001; Gałecki et al., 2009). It may be prudent in future studies to carry out an assessment of MDA after variable time spent crib biting, as this may yield results that are consistent with the human literature. For example, if crib biting lowers the levels of antioxidant enzymes, it would be predicted that long-term crib biters would show elevated levels of MDA relative to more novice crib biters. Unfortunately, accurate information regarding the amount of time crib biting in the present sample was not available.

Finally, crib-biting is complex and multifactorial, and its causal factors and function are still not well understood. However, one line of research has suggested that stereotypies such as crib-biting are the result of neurological changes in response to the animal experiencing repeated failed attempts to cope with environmental challenges (McBride and Parker, 2015; McGreevy and Nicol, 1998; McGreevy et al., 1995; Parker et al., 2008b). Stressinduced changes in mesoaccumbens dopamine neurophysiology, for example, have been associated with the development of stereotypic behavior in in-bred strains of laboratory rodents (Cabib et al., 1998), and similar changes were observed in crib-biting horses (McBride and Hemmings, 2005). Specifically, crib biting horses have increased activity within the mesoaccumbens dopamine pathway as evidenced by increased D1 and D2-type dopamine receptors in the nucleus accumbens and dorsal striatum. Interestingly, the pathophysiology of two neurological conditions that are strongly associated with dysfunction of brain dopamine levels, the neurodegenerative disease Parkinson's and the neuropsychiatric disorder, schizophrenia, has been linked to oxidative stress. Patients with Parkinson's disease have a selective loss of dopamine neurons in the substantia nigra and subsequently decreased dopamine levels in the striatum regions of in the brain. Post-mortem studies of patients with Parkinson's disease have revealed high levels of MDA in the substantia nigra, the primary site of dopamine cell loss in the disease (Dexter et al., 1989). In addition, Parkinson's patients have been shown to have increased levels of SOD in the same region (Marttila et al., 1988). Schizophrenic patients have an overexpression of D2 dopamine receptors in the mesocorticolimbic dopamine pathways (Abi-Dargham et al., 2000) and schizophrenic patients were observed to have elevated SOD (Abdalla et al., 1986). However, in another neuropsychiatric disorder linked to dopamine, attention-deficit hyperactivity disorder (ADHD), patients were found to have significantly lower SOD (Selek et al., 2008) as were patients with depressive disorders (Galeki et al., 
2009). Although very complex and beyond the scope of this paper, dopamine, and indeed other catecholamine, metabolism results in the production of many ROS (see Meiser, 2013 for a recent review). Thus, generally higher levels of midbrain dopamine, as is observed in crib biters, would be expected to result in increased oxidative stress in these regions. When this is considered in line with our current findings, it suggests the intriguing hypothesis that alterations in dopamine neurochemistry and subsequent increased dopamine metabolism may be associated with tissue damage involving free radicals in the brain of crib-biters as is the case in humans with psychiatric disorder (Evans, 1993).

\section{Conclusions}

Based on our observations, we suggest that the antioxidant system is impaired during an acute crib-biting episode, and generally lower antioxidant defenses against lipid peroxidation exist in horses display crib-biting. Although it is clear that oxidative stress is correlated with crib-biting, in order to understand the direction of causation, further studies will be necessary. For example, it may be that a third factor (e.g., HPA response to psychological stress) is involved both in increasing crib biting and decreasing antioxidant enzymes. However, out findings also raise the possibility that oxidative stress is involved in the pathophysiology of crib biting. Although this will require further investigation, our findings have identified a novel biomarker for crib biting. Indeed, it may be that decreases in antioxidant enzymes might be used as an early marker/risk factor for the development of crib biting. This would be extremely useful for owners and veterinarians in order to ensure that any preventative/early interventions are put into place for at-risk individuals. Finally, our findings provide theoretical basis for the development of novel therapeutic strategies for crib-biting horses such as antioxidant supplementation, even if this is in a palliative capacity.

\section{Acknowledgments}

The authors gratefully acknowledge the financial support for this work provided by the Vice Chancellor for Research at Shiraz University.

\section{Authorship}

The idea for the paper was conceived by A. O; Authors A. O. and S. V. developed the research, and managed the literature searches, M. O. P. undertook the statistical analyses, A. O and M. O. P. wrote and approved the final 
manuscript. S. N. measured the laboratory parameters.

\section{Conflict of interest}

The authors declare that they have no conflict of interest.

\section{References}

Abdalla, D. S., Monteiro, H. P., Oliveira, J. A., Bechara, E. J., 1986. Activities of superoxide dismutase and glutathione peroxidase in schizophrenic and manic-depressive patients. Clinical Chemistry, 32, 805-807.

Abi-Dargham, A., et al., 2000. Increased baseline occupancy of D2 receptors by dopamine in schizophrenia. PNAS, 97, 8104-8109.

Andersen, J. K., 2004. Oxidative stress in neurodegeneration: cause or consequence?. Nature Reviews Neuroscience, 5, S18-S25.

Archer, D. C., Freeman, D. E., Doyle, A. J., Proudman, C. J., Edwards, G. B., 2004. Association between cribbing and entrapment of the small intestine in the epiploic foramen in horses: 68 cases (1991-2002). JAVMA, 224, 562564.

Armstrong, D. and Browne, R., 1994. The analysis of free radicals, lipid peroxides, antioxidant enzymes and compounds related to oxidative stress as applied to the clinical chemistry laboratory. In: Free radicals in diagnostic medicine (pp. 43-58). Springer US.

Bachmann, I., Audige, L., Stauffacher, M., 2003. Risk factors associated with behavioural disorders of crib-biting, weaving and box-walking in Swiss horses. Equine Vet. J. 35, 158-163.

Bilici, M., Efe, H., Köroğlu, M. A., Uydu, H. A., Bekaroğlu, M., Değer, O., 2001. Antioxidative enzyme activities and lipid peroxidation in major depression: alterations by antidepressant treatments. J. Affect. Disord. 64, 43-51.

Bouayed, J., Rammal, H., Soulimani, R., 2009. Oxidative stress and anxiety: relationship and cellular pathways. Oxid. Med. Cell Longev. $2,63-67$.

Cabib, S., Giardino, L., Calza, L., Zanni, M., Mele, A., Puglisi-Allegra, S., 1998. Stress promotes major changes in dopamine receptor densities within the mesoaccumbens and nigrostriatal systems. Neuroscience 84, 193-200.

Ceciliani, F., Giordano, A., Spagnolo, V., 2002. The systemic reaction during inflammation: the acute-phase proteins. Protein Pept. Lett. 9, 211-223.

Cooper, J. J., Albentosa, M. J., 2005. Behavioural adaptation in the domestic horse: potential role of apparently abnormal responses including stereotypic behaviour. Livest. Prod. Sci. 92, 177-182.

Cooper, J., McGreevy, P., 2007. Stereotypic behaviour in the stabled horse: causes, effects and prevention without compromising horse welfare. In The welfare of horses (pp. 99-124). Springer Netherlands.

Cumurcu, B. E., Ozyurt, H., Etikan, I., Demir, S., Karlidag, R., 2009. Total antioxidant capacity and total oxidant status in patients with major depression: impact of antidepressant treatment. P Py chiatry Clin. Neurosci. 63, 639-645. 
Dadheech, G., Mishra, S., Gautam, S., Sharma, P., 2006. Oxidative stress, $\alpha$-tocopherol, ascorbic acid and reduced glutathione status in schizophrenics. Indian J. Clin. Biochem. 21, 34-38.

Dexter, D.T. et al., 1989. Basal lipid peroxidation in substantia nigra is increased in Parkinson's disease. J. Neurochem. 52, 381-389.

Eckersall P. D., Bell R., 2010. Acute phase proteins: Biomarkers of infection and inflammation in veterinary medicine. Vet. J. 185(1): 23-27.

Erel, O., 2004. A novel automated direct measurement method for total antioxidant capacity using a new generation, more stable ABTS radical cation. Clinical biochemistry, 37: 277-285.

Evans, P. H., (1993). Free radicals in brain metabolism and pathology. Br. Med. J. 49, 577-587.

Fournier T., Medjoubi-N N., Porquet D., 2000. Alpha-1-acid glycoprotein. Biochim. Biophys. Acta. - Prot. Struct. Mol. Enzymol. 1482: 157-171.

Freymond, S. B., Bardou, D., Briefer, E., Bruckmaier, R., Fouché, N., Fleury, J., Bachmann, I., 2015. The physiological consequences of crib-biting in horses in response to an ACTH challenge test. Physiol. Behav. 151, 121-128.

Gałecki, P., Szemraj, J., Bieńkiewicz, M., Florkowski, A., Gałecka, E., 2009. Lipid peroxidation and antioxidant protection in patients during acute depressive episodes and in remission after fluoxetine treatment. Pharmacol. Rep. 61, $436-447$.

Halliwell, B., Gutteridge, J. M., 1990. The antioxidants of human extracellular fluids. Archives of biochemistry and biophysics, 280, 1-8.

Hillyer, M.H., Taylor, F.G.R., Proudman, C.J., Edwards, G.B., Smith, J.E., French, N.P., 2002. Case control study to identify risk factors for simple colonic obstruction and distension colic in horses. Equine Vet. J., 34, 455-463.

Jones, K. A., Thomsen, C., 2013. The role of the innate immune system in psychiatric disorders. Mol. Cell. Neurosci. 53, 5262 .

Love, S., 1999. Oxidative stress in brain ischemia. Brain Pathology, 9, 119-131.

Luescher, U. A., McKeown, D. B., \& Dean, H., 1998. A cross-sectional study on compulsive behaviour (stable vices) in horses. Equine Vet. J. 30, Suppl. 27, 14-18.

Lykkesfeldt, J., 2001. Determination of Malondialdehyde as dithiobarbituric acid adduct in biological samples by HPLC with fluorescence detection: Comparison with ultraviolet-visible spectrophotometry. Clin. Chem. 47: 1725-1727.

Maes, M., Galecki, P., Chang, Y. S., Berk, M., 2011. A review on the oxidative and nitrosative stress (O\&NS)

pathways in major depression and their possible contribution to the (neuro) degenerative processes in that illness. Prog.

Neuropsychopharmacol. Biol. Psychiatry. 35, 676-692.

Marttila, R. J., Lorentz, H., Rinne, U. K., 1988. Oxygen toxicity protecting enzymes in Parkinson's disease: increase of superoxide dismutase-like activity in the substantia nigra and basal nucleus. Journal of the neurological sciences, 86 , 321-331.

Maurya, P. K., Noto, C., Rizzo, L. B., Rios, A. C., Nunes, S. O., Barbosa, D. S., Brietzke, E., 2016. The role of oxidative and nitrosative stress in accelerated aging and major depressive disorder. Prog. Neuropsychopharmacol. Biol. Psychiatry. 65, 134-144.

McBride, S. D., Hemmings, A., 2005. Altered mesoaccumbens and nigro-striatal dopamine physiology is associated with stereotypy development in a non-rodent species. Behav. Brain Res. 159, 113-118.

McBride, S., Hemmings, A., 2009. A neurologic perspective of equine stereotypy. J. Equine Vet. Sci. 29, 10-16. McBride, S. D., Parker, M. O., 2015. The disrupted basal ganglia and behavioural control: An integrative cross- domain perspective of spontaneous stereotypy. Behav. Brain Res. 276, 45-58.

McBride, S., Cuddeford, D., 2001. The putative welfare-reducing effects of preventing equine stereotypic behaviour. Anim. 
Welf. 10, 173-189.

McGreevy, P., Nicol, C. (1998). Physiological and behavioral consequences associated with short-term prevention of cribbiting in horses. Physiol. Behav. 65, 15-23.

McGreevy, P., Cripps, P., French, N., Green, L., Nicol, C. J., 1995. Management factors associated with stereotypic and redirected behaviour in the Thoroughbred horse. Equine Vet. J. 27, 86-91.

McGreevy, P. D., Richardson, J. D., Nicol, C. J., Lane, J. G., 1995. Radiographic and endoscopic study of horses performing an oral based stereotypy. Equine Vet. J., 27, 92-95.

Miller, A. H., Raison, C. L., 2016. The role of inflammation in depression: from evolutionary imperative to modern treatment target. Nat. Rev. Immunol. 16, 22-34.

Moeller, B.A., McCall, C.A., Silverman, S.J. and McElhenney, W.H., 2008. Estimation of saliva production in crib-biting and normal horses. Journal of Equine Veterinary Science, 28(2), pp.85-90.

Murata H., Shimada N., Yoshioka M., 2004. Current research on acute phase proteins in veterinary diagnosis: an overview. Vet. J. 168: 28-40

Nagy, K., Bodo, G., Bárdos, G., Banszky, N., Kabai, P., 2010. Differences in temperament traits between crib- biting and control horses. Appl. Anim. Behav. Sci. 122, 41-47.

Ng, F., Berk, M., Dean, O., Bush, A. I., 2008. Oxidative stress in psychiatric disorders: evidence base and therapeutic implications. Int. J. Neuropsychopharmacol. 11, 851-876.

Nicol, C.J., Davidson, H.P., Harris, P.A., Waters, A.J. Wilson, A.D., 2002. Study of crib-biting and gastric inflammation and ulceration in young horses. The Veterinary Record, 151(22), pp.658-662.

Ozcan, M. E., Gulec, M., Ozerol, E., Polat, R., Akyol, O., 2004. Antioxidant enzyme activities and oxidative stress in affective disorders. Int. Clin. Psychopharmacol. 19, 89-95.

Pace, T. W., Mletzko, T. C., Alagbe, O., Musselman, D. L., Nemeroff, C. B., Miller, A. H., Heim, C. M., 2006. Increased stress-induced inflammatory responses in male patients with major depression and increased early life stress. Am. J. Psychiatry. 163, 1630-1633.

Paglia, D. E., Valentine, W. N., 1967. Studies on the quantitative and qualitative characterization of erythrocyte glutathione peroxidase. J. Lab. Clin. Med. 70, 158-169.

Parker, M., Redhead, E. S., Goodwin, D., McBride, S. D., 2008a. Impaired instrumental choice in crib-biting horses (Equus caballus). Behav. Brain Res. 191, 137-140.

Parker, M., Goodwin, D., Redhead, E. S., 2008b. Survey of breeders' management of horses in Europe, North America and Australia: comparison of factors associated with the development of abnormal behaviour. Appl. Anim. Behavi. Sci. 114, 206215 .

Parker, M., McBride, S. D., Redhead, E. S., Goodwin, D., 2009. Differential place and response learning in horses displaying an oral stereotypy. Behav. Brain Res. 200, 100-105.

Raison, C. L., Miller, A. H., 2011. Is depression an inflammatory disorder?. Curr. Psychiatry. Rep. 13, 467-475.

Raison, C. L., Capuron, L., Miller, A. H., 2006. Cytokines sing the blues: inflammation and the pathogenesis of depression. Trends Immunol. 27, 24-31.

Sarandol, A., Sarandol, E., Eker, S. S., Erdinc, S., Vatansever, E., \& Kirli, S., 2007. Major depressive disorder is accompanied with oxidative stress: short-term antidepressant treatment does not alter oxidative-antioxidative systems. Hum. Psychopharm. Clin. 22, 67-73. 
Sarrafchi, A., Blokhuis, H. J., 2013. Equine stereotypic behaviors: causation, occurrence, and prevention. J. Vet. Behav. 85, 386-394.

Selek, S., Savas, H. A., Gergerlioglu, H. S., Bulut, M., Yilmaz, H. R., 2008. Oxidative imbalance in adult attention deficit/hyperactivity disorder. Biological psychology, 79, 256-259.

Serafini, M., Del Rio D., 2004. Understanding the association between dietary antioxidants, redox status and disease: is the total antioxidant capacity the right tool? Redox Rep. 93, 145-152.

Sharma, R.K., Pasqualotto, F.F., Nelson, D.R., Thomas, A.J. and Agarwal, A., 1999. The reactive oxygen species-total antioxidant capacity score is a new measure of oxidative stress to predict male infertility. Human Reproduction, 14(11), pp.2801-2807.

Slaughter, M., R, O’brien, P. J., 2000. Fully-automated spectrophotometric method for measurement of antioxidant activity of catalase. Clin. Biochem. 33, 525-534.

Smith, C. D., Carney, J. M., Starke-Reed, P. E., Oliver, C. N., Stadtman, E. R., Floyd, R. A., Markesbery, W. R., 1991. Excess brain protein oxidation and enzyme dysfunction in normal aging and in Alzheimer disease. PNAS, 88, 10540-10543.

Wickens, D.G., Norden, A.G., Lunec, J. and Dormandy, T.L., 1983. Fluorescence changes in human gamma-globulin induced by freeradical activity. Biochimica et Biophysica Acta (BBA)-Protein Structure and Molecular Enzymology, 742(3), pp.607-616.

Witko-Sarsat, V., Friedlander, M., Capeillère-Blandin, C., Nguyen-Khoa, T., Nguyen, A.T., Zingraff, J., Jungers, P. and DescampsLatscha, B., 1996. Advanced oxidation protein products as a novel marker of oxidative stress in uremia. Kidney international, 49, 13041313. 
Table 1: Demographic and breed characteristics of crib biting and control horses

\begin{tabular}{|c|c|c|c|c|c|}
\hline \multicolumn{3}{|l|}{ Crib biters } & \multicolumn{3}{|l|}{ Control } \\
\hline Age (years) & Sex & Breed & Age (years) & Sex & Breed \\
\hline 14 & $\mathrm{~m}$ & Dareshouri-Arab & 10 & $\mathrm{~m}$ & Dareshouri-Arab \\
\hline 12 & gelding & Throroughbred-Turkoman & 12 & gelding & Throroughbred-Turkoman \\
\hline 12 & $\mathrm{~m}$ & Dareshouri-Arab & 9 & $\mathrm{~m}$ & Dareshouri-Arab \\
\hline 9 & $f$ & Arab & 9 & $f$ & Arab \\
\hline 10 & $\mathrm{~m}$ & Dareshouri-Arab & 12 & $\mathrm{~m}$ & Dareshouri-Arab \\
\hline 3 & $f$ & Dareshouri & 8 & $f$ & Dareshouri \\
\hline 7 & $\mathrm{~m}$ & Turkoman & 7 & $\mathrm{~m}$ & Turkoman \\
\hline 2 & $\mathrm{~m}$ & Arab & 3 & $\mathrm{~m}$ & Dareshouri \\
\hline 6 & $\mathrm{~m}$ & Dareshouri & 2 & $\mathrm{~m}$ & Dareshouri-Arab \\
\hline 9 & $\mathrm{~m}$ & Dareshouri-Arab & 9 & $\mathrm{~m}$ & Dareshouri-Arab \\
\hline
\end{tabular}


Table 2: Summary statistics for vital signs and age in control, crib-biters [basal] and crib-biters [acute crib-biting] horses.

\begin{tabular}{ccccc}
\hline Variable & Control & Basal & $\begin{array}{c}\text { Acute Crib- } \\
\text { Biting }\end{array}$ & $P(\mathrm{LMM})$ \\
\hline $\begin{array}{c}\text { HR }(\mathrm{BPM}) \\
\begin{array}{c}\text { Temperature } \\
\left({ }^{\circ} \mathrm{C}\right)\end{array}\end{array}$ & $34 \pm 5.83$ & $35 \pm 5.45$ & $34.4 \pm 6.32$ & 0.93 \\
$\begin{array}{c}\text { Respiratory } \\
\text { rates }\end{array}$ & $18.70 \pm 3.23$ & $17.70 \pm 4.78$ & $19.10 \pm 3.41$ & 0.86 \\
$($ breaths/min) & & $36.96 \pm 0.59$ & $37.06 \pm 0.70$ & 0.71 \\
Age (years) & $8.20 \pm 3.39$ & $8.40 \pm 3.92$ & $8.40 \pm 3.92$ & 0.99 \\
\hline
\end{tabular}


Table 3: Evaluated physiological parameters in control, crib-biters (basal) and crib-biters (cribbing) horses

\begin{tabular}{ccccc}
\hline Variable & Control & Basal & $\begin{array}{c}\text { Acute Crib- } \\
\text { Biting }\end{array}$ & $\boldsymbol{P}(\mathbf{L M M})$ \\
\hline TP $(\mathrm{g} / \mathrm{dl})$ & $6.42 \pm 0.64^{\mathrm{a}}$ & $6.63 \pm 0.44^{\mathrm{a}}$ & $6.88 \pm 0.4^{\mathrm{a}}$ & 0.76 \\
$\mathrm{ALB}(\mathrm{g} / \mathrm{dl})$ & $3.39 \pm 0.15^{\mathrm{a}}$ & $3.44 \pm 0.19^{\mathrm{a}}$ & $3.39 \pm 0.28^{\mathrm{a}}$ & 0.98 \\
GLB $(\mathrm{g} / \mathrm{dl})$ & $3.02 \pm 0.56^{\mathrm{a}}$ & $3.19 \pm 0.41^{\mathrm{a}}$ & $3.49 \pm 0.55^{\mathrm{a}}$ & 0.75 \\
$\mathrm{SAA}(\mu \mathrm{g} / \mathrm{ml})$ & $7.27 \pm 0.41^{\mathrm{a}}$ & $8.65 \pm 0.76^{\mathrm{a}}$ & $9.02 \pm 0.89^{\mathrm{a}}$ & 0.14 \\
AGP $(\mathrm{pg} / \mathrm{ml})$ & $96.23 \pm 5.07^{\mathrm{a}}$ & $96.32 \pm 5.11^{\mathrm{a}}$ & $98.03 \pm 3.83^{\mathrm{a}}$ & 0.96 \\
$\mathrm{Hp}(\mathrm{ng} / \mathrm{ml})$ & $3.61 \pm 0.13^{\mathrm{a}}$ & $3.59 \pm 0.15^{\mathrm{a}}$ & $3.5 \pm 0.15^{\mathrm{a}}$ & 0.87 \\
$\mathrm{MDA}(\mathrm{mmol} / \mathrm{l})$ & $1.27 \pm 0.036^{\mathrm{a}}$ & $1.34 \pm 0.048^{\mathrm{a}}$ & $1.41 \pm 0.05^{\mathrm{a}}$ & 0.10 \\
TAC $(\mathrm{mmol} / \mathrm{l})$ & $0.81 \pm 0.0071^{\mathrm{a}}$ & $0.75 \pm 0.0084^{\mathrm{b}}$ & $0.7 \pm 0.0094^{\mathrm{c}}$ & $<0.001$ \\
SOD $(\mathrm{u} / \mathrm{gHb})$ & $562.8 \pm 7.32^{\mathrm{a}}$ & $506.2 \pm 3.64^{\mathrm{b}}$ & $450.8 \pm 4.37^{\mathrm{c}}$ & $<0.001$ \\
GPx $(\mathrm{u} / \mathrm{gHb})$ & $58.2 \pm 1.73^{\mathrm{a}}$ & $50.4 \pm 1.17^{\mathrm{b}}$ & $42.9 \pm 0.82^{\mathrm{c}}$ & $<0.001$ \\
CAT $(\mathrm{u} / \mathrm{gHb})$ & $174.5 \pm 2.47^{\mathrm{a}}$ & $162.9 \pm 1.68^{\mathrm{b}}$ & $129.9 \pm 1.81^{\mathrm{c}}$ & $<0.001$ \\
\hline
\end{tabular}

TP: Total protein; ALB: Albumin; GLB; Globulin; SAA: Serum amyloid-A; AGP: Alpha -1- acid glycoprotein; Hp: Haptoglobin; MDA: Malondialdehyde; TAC: Total antioxidant capacity; SOD: Superoxide dismutase; GPx: Glutathione peroxidase; CAT: Catalase. Note: For each variable, shared letters indicate post-hoc analyses not significantly different. Different letters indicate $P<0.01$ 
Table 4: Intercorrelations (Pearson's) between evaluated physiological parameters.

\begin{tabular}{|c|c|c|c|c|c|c|c|c|c|c|}
\hline & 1 & 2 & 3 & 4 & 5 & 6 & 7 & 8 & 9 & 10 \\
\hline 1. TP & - & & & & & & & & & \\
\hline 2. ALB & 0.195 & - & & & & & & & & \\
\hline 3. GLB & $.910 * *$ & -0.229 & - & & & & & & & \\
\hline 4. SAA & -0.073 & -0.133 & -0.016 & - & & & & & & \\
\hline 5. AGP & $.397 *$ & 0.037 & $.379 *$ & 0.011 & - & & & & & \\
\hline 6. HP & 0.058 & 0.25 & -0.048 & 0.005 & -0.209 & - & & & & \\
\hline 7. MDA & 0.064 & 0.079 & 0.031 & -0.358 & 0.234 & 0.025 & - & & & \\
\hline 8. TAC & -0.199 & -0.055 & -0.175 & $-.369 *$ & -0.131 & 0.1 & -0.261 & - & & \\
\hline 9. SOD & -0.152 & 0.11 & -0.197 & -0.316 & 0.032 & 0.189 & -0.32 & $.809 * *$ & - & \\
\hline 10. GPx & -0.066 & 0.007 & -0.069 & -0.239 & -0.058 & 0.163 & -0.179 & $.762 * *$ & $.773 * *$ & - \\
\hline 11. CAT & -0.047 & 0.092 & -0.086 & -0.328 & 0.009 & 0.058 & -0.305 & $.801 * *$ & $.883 * *$ & .761 \\
\hline
\end{tabular}

TP: Total protein; ALB: Albumin; GLB; Globulin; SAA: Serum amyloid-A; AGP: Alpha -1- acid glycoprotein; Hp: Haptoglobin; MDA: Malondialdehyde; TAC: Total antioxidant capacity; SOD: Superoxide dismutase; GPx: Glutathione peroxidase; CAT: Catalase. Note: $* *$ Correlation is significant at the 0.01 level (2-tailed); ${ }^{*}$ Correlation is significant at the 0.05 level (2-tailed). 